\title{
An Optical Method for the Determination of the Cholesteric Pitch in Liquid Crystals
}

\author{
Ingo Dierking, Frank Gießelmann, and Peter Zugenmaier \\ Institut für Physikalische Chemie der TU Clausthal, Arnold-Sommerfeld-Straße 4, \\ D-38678 Clausthal-Zellerfeld.
}

Z. Naturforsch. 50a, 589-594 (1995); received December 17, 1994

\begin{abstract}
Preparation of a cholesteric liquid crystalline phase in planar orienting liquid crystal cells exhibits discontinuous colour changes with temperature when viewed between crossed polarizers. The transmission spectra of such a system can be described by a modified de Vries equation, with the birefringence dispersion included in a simplified single-band model. With a suitable choice of the cell gap as compared to the cholesteric pitch, the procedure can be used as a new method to determine the temperature dependence of the cholesteric pitch.
\end{abstract}

Key words: Liquid crystal, cholesteric mesophase, pitch determination, birefringence dispersion, twist inversion.

\section{Introduction}

The cholesteric or chiral nematic liquid crystalline phase exhibits locally a nematic director configuration which is continuously twisted in a direction perpendicular to the local nematic director $\boldsymbol{n}$, thus forming macroscopically a helical structure. The spatial period of this structure is given by one-half its pitch $P$, that is a $\pi$-twist, as the $\boldsymbol{n}$ and $-\boldsymbol{n}$ directions are equivalent (headtail symmetry). The optical axis is directed along the axis of the cholesteric helix. If the $z$-axis is chosen as the helical axis, the spatial director configuration $\boldsymbol{n}(\boldsymbol{r})$ of the ideal cholesteric structure is described by

$$
\begin{aligned}
& n_{x}=\cos \left(\frac{2 \pi}{P} z+\phi_{0}\right), \\
& n_{y}=\sin \left(\frac{2 \pi}{P} z+\phi_{0}\right), \\
& n_{z}=0,
\end{aligned}
$$

where $P$ is the cholesteric pitch, and the value of the phase angle $\phi_{0}$ is arbitrary (depending on the boundary conditions). Both the magnitude and sign of $P$ are important. The sign describes the handedness of the helicoidal structure (negative for a left- and positive for a right-handed screw), and the magnitude of the cholesteric pitch generally changes with temperature.

Reprint requests to I. Dierking.

\section{Theoretical}

Due to its helical structure, the cholesteric phase possesses unique optical properties such as a very high rotatory power along its optical axis in the order of several thousand degrees per millimeter, a selective reflexion of one component of circular polarized light of specific wavelength, and an anomalous behaviour of the rotatory dispersion in the neighbourhood of the region of selective reflexion. The optical properties of cholesteric phases were described theoretically by de Vries [1] on the basis of anisotropic layers which form a helical structure. He derived an equation to describe the optical rotatory power $\varphi$ as a function of wavelength $\lambda$ in radians per unit length:

$$
\varphi=\frac{\pi \Delta n^{2} P}{4 \lambda^{2}} \frac{1}{1-\left(\frac{\lambda}{\lambda_{0}}\right)^{2}}
$$

with $\lambda_{0}$ being the wavelength of selective reflexion which is related to the pitch by $\lambda_{0}=\bar{n} P$, and with $\bar{n}$ the average nematic index of refraction for a nematic layer. The birefringence $\Delta n$ has to be described as a function of wavelength.

Starting from the Vuks-equation [2], which correlates the microscopic molecular polarizabilities of an optically anisotropic, uniaxial liquid crystal to the macroscopic refractive indices, Wu has derived a semiempirical model to describe the birefringence dispersion of a liquid crystal [3-6]. In a simplified singleband approximation, the temperature and wavelength 
dependence of the birefringence is described by [3]

$$
\Delta n(T, \lambda)=G(T) \frac{\lambda^{2}\left(\lambda^{*}\right)^{2}}{\lambda^{2}-\left(\lambda^{*}\right)^{2}},
$$

where $\lambda^{*}$ is a mean resonance vacuum wavelength of the first UV absorption band and $G(T)$ contains terms like the number of molecules per unit volume, the nematic order parameter $S$ and the effective quantummechanical oscillator strengths. $G(T)$ relies in first approximation on the temperature dependence of the order parameter $S$, which varies between $S=1$ (perfect nematic order) and $S=0$ (isotropic order) according to the Maier-Saupe-theory [7, 8].

Inserting (3) into (2) and accounting for the cell gap $d$ (the sample thickness), a modified de Vries-equation is obtained for the optical rotation $\alpha$ :

$$
\alpha=\varphi d=\frac{\pi P}{4 \lambda^{2}}\left[G \frac{\lambda^{2}\left(\lambda^{*}\right)^{2}}{\lambda^{2}-\left(\lambda^{*}\right)^{2}}\right]^{2} \frac{1}{1-\left(\frac{\lambda}{\bar{n} P}\right)^{2}} d .
$$

The normalized transmitted intensity $I$ (in per cent) between crossed polarizers as a function of wavelength is given by

$$
I(\lambda)=100 \sin ^{2}(\alpha)
$$

As the pitch is temperature dependent, the normalized transmitted intensity of a planar oriented cholesteric phase of sample thickness $d$ can then be written as (inserting (4) into (5)):

$$
\begin{aligned}
I(T, \lambda) & =100 \sin ^{2} \\
\cdot & {\left[\frac{\pi P(T)}{4 \lambda^{2}}\left[G(T) \frac{\lambda^{2}\left(\lambda^{*}\right)^{2}}{\lambda^{2}-\left(\lambda^{*}\right)^{2}}\right]^{2} \frac{1}{1-\left(\frac{\lambda}{\bar{n} P(T)}\right)^{2}} d\right] . }
\end{aligned}
$$

A graphic representation of this expression is depicted in Fig. 1 as a function of $\lambda$ for a range of the cholesteric pitch of $1-10 \mu \mathrm{m}$ and a cell gap of $10 \mu \mathrm{m}$. Maxima of transmitted intensity are clearly observable, and the colour observed in the microscope between crossed polarizers is determined by (6).

\section{Experimental}

The benzoic acid biphenyl ester

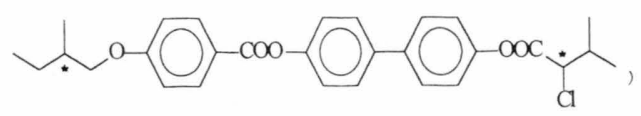

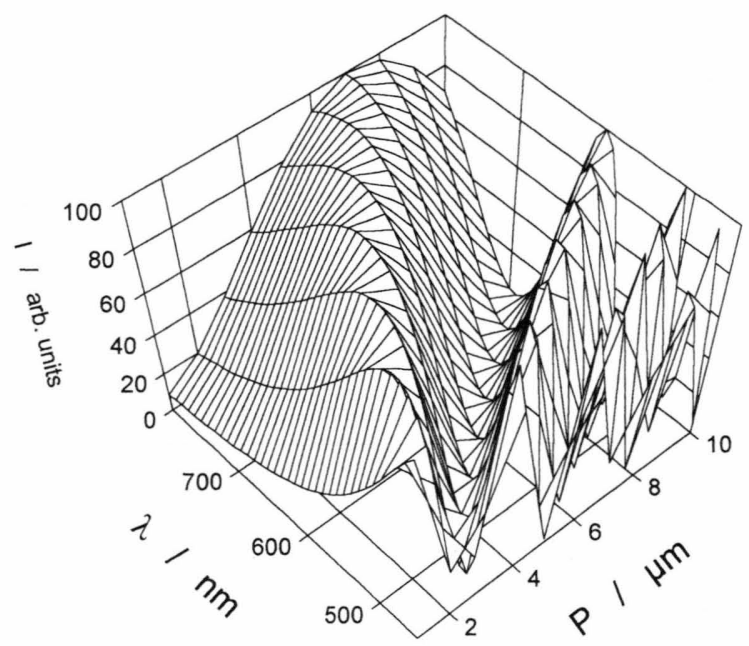

Fig. 1. Graphical representation of (6) to illustrate the shift of maxima and minima of transmitted light intensity between crossed polarizers as a function of wavelength and pitch, i.,e. temperature, calculated for a $10 \mu \mathrm{m}$ cell.

abbreviated as (S, S) M96, exhibits a rarely observed inversion of the sign of $P$ with temperature for single component systems, changing the handedness of the helicoidal structure. Such a cholesteric twist inversion is characterized by a diverging pitch on approaching a specific inversion temperature $T_{\text {inv }}$. The advantage of using the above compound is the wide range of values of the cholesteric pitch from $P=1 \mu \mathrm{m}$ at temperatures close to the clearing point to $P \rightarrow \infty$ as $T \rightarrow T_{\text {inv }}$. The synthesis, characterization and properties of this compound are reproted in [9-11]. The phase sequence on cooling is

$$
\text { I } 163 \mathrm{~N}^{*} 105.3 \mathrm{~S}_{\mathrm{C}}^{*} 76.4 \mathrm{~S}_{\mathrm{G}}^{*} 30 \text { Cryst. }
$$

with the twist inversion temperature $T_{\text {inv }}=114^{\circ} \mathrm{C}$. The higher ordered crystalline smectic phase $\left(\mathrm{S}_{\mathrm{G}}^{*}\right)$ was identified by texture observation, electrooptic experiments and miscibility studies with the $n=4$ homologue of the extensively investigated TBAA series [12$15]$ as reference compound.

In planar orienting liquid crystal cells (commercially available from E.H.C. Ltd. Japan), a very thin polyimide layer is brought onto the glass plates (spin coating) and rubbed in one direction, thus imposing boundary conditions on the cholesteric system. The molecular orientation at the bottom and the top of the cell are fixed and parallel (Figure 2). To minimize the distortion energy, the system chooses an integral number $Z=1,2,3 \ldots$ of half-pitches $P / 2$ to fit into the cell 


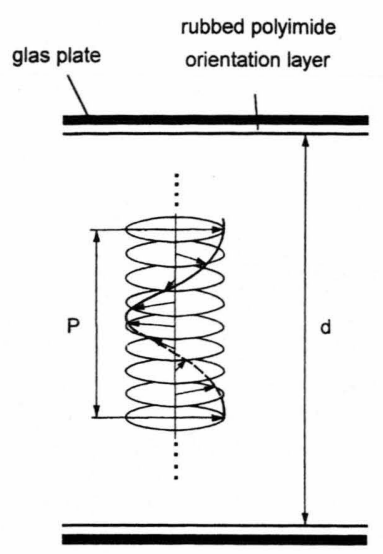

Fig. 2. Schematic illustration of the geometry used for the investigations. The rubbed polyimide orientation layer imposes boundary conditions on the cholesteric system. The molecular orientation at the bottom and top of the cell are fixed. In the cell gap $d$ an integral number of half-pitches $Z=\mathrm{d} /(P / 2)$, i.e. $\pi$-twists, is observed.

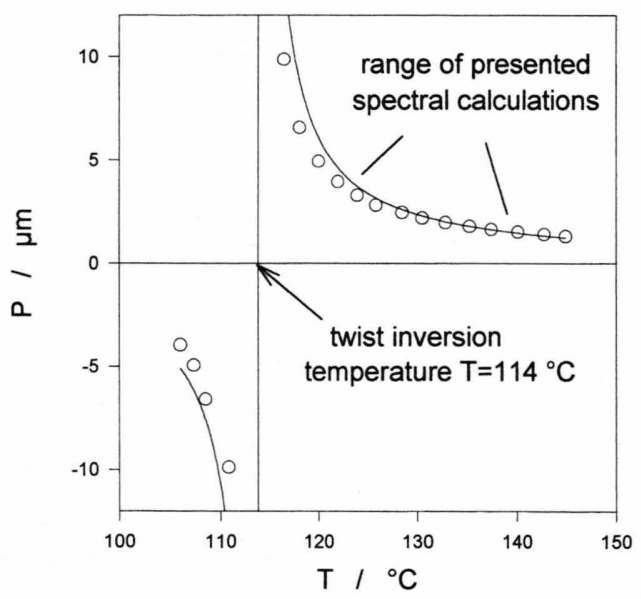

Fig. 3. Temperature dependence of the cholesteric pitch $P$, determined with the colour change method in a $10 \mu \mathrm{m}$ planar orienting liquid crystal cell.

gap, i.e. $Z=d /(P / 2)$, if the pitch is not too large $(P \approx d)$. This property was used by Cano [16] and Grandjean [17] to determine the cholesteric pitch of samples prepared between a glass plate and a lense of known radius or a wedge. For systems exhibiting a cholesteric twist inversion with temperature variation, this property can be used to adjust an integral number of halfpitches, $P / 2,2 P / 2,3 P / 2 \ldots$ in the cell of given thickness $d$, depending on the temperature. At the twist inversion temperature $T_{\text {inv }}$, the cholesteric pitch diverges and $P / 2 \gg d$, so that a nematic director configuration is observed. Increasing the temperature, the cholesteric pitch decreases until the condition $P(T) / 2=d$ is satisfied. At this point a discontinuous colour change is observed as the structure changes from $Z=0$ to $Z=1$. Further increase of the temperature eventually results in another discontinuous colour change when the condition $2 P(T) / 2=d$ is fulfilled and the structure changes from $Z=1$ to $Z=2$. This behaviour continues as the temperature is increased, and a definite number of half-pitches can be adjusted with $Z=1,2,3, \ldots$. It should be noted that the variation of temperature

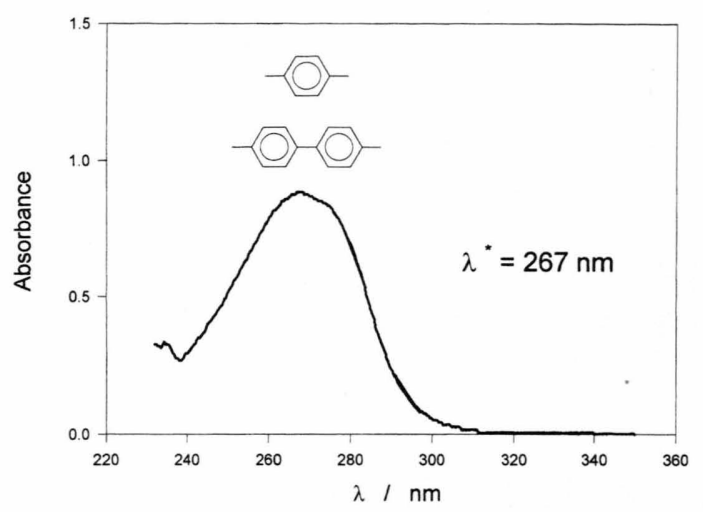

Fig. 4. UV spectrum of the compound (S,S)M96 dissolved in chloroform to determine the mean resonance wavelength of the first UV absorption band $\lambda^{*}$.

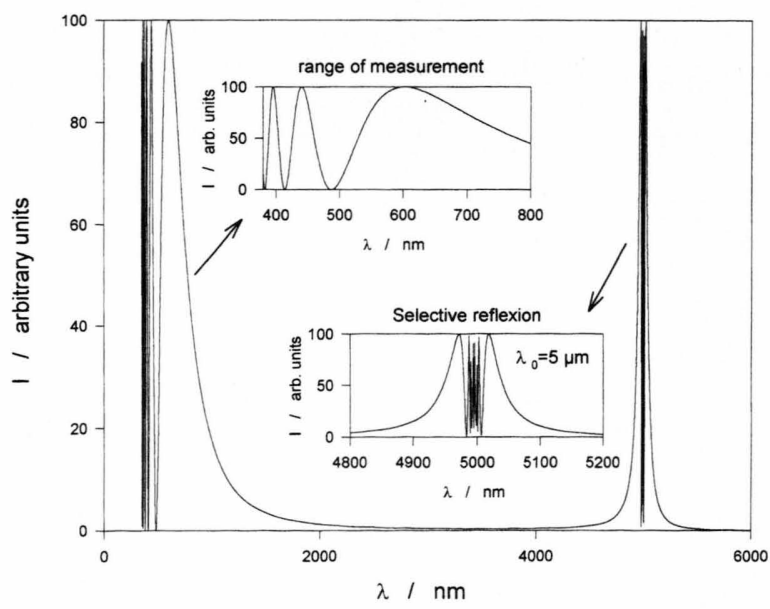

Fig. 5. Normalized transmitted intensity between crossed polarizers as a function of wavelength $\lambda$, calculated according to (6) for a selective reflexion $\lambda_{0}=5000 \mathrm{~nm}$. The curve depicts the range of measurement $(350-800 \mathrm{~nm})$, far away from the selective reflexion, as typical experimental conditions. 

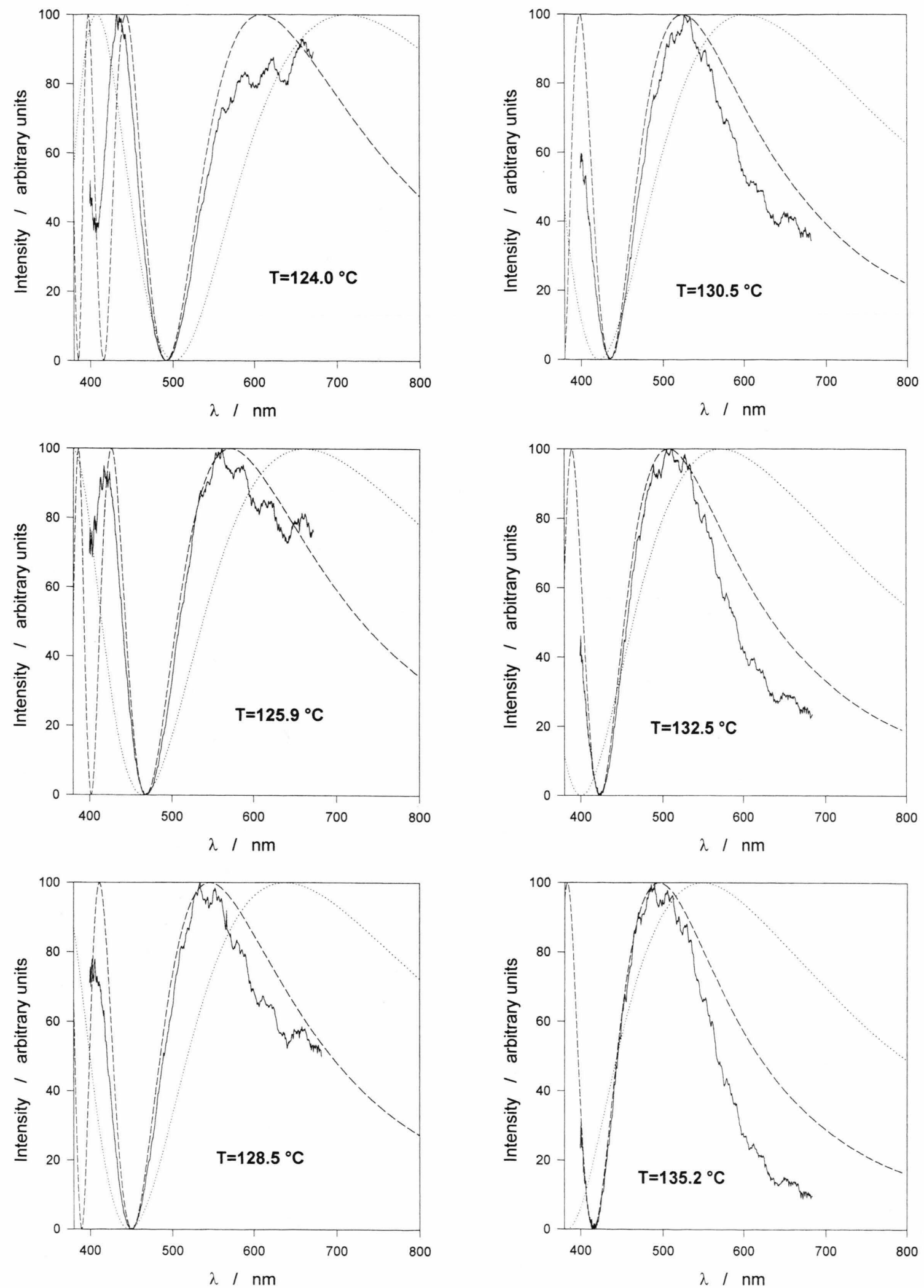

Fig. 6. Normalized experimental transmission spectra between crossed polarizers for different temperatures i.e. different twist states $Z$. (a) $T=124.0^{\circ} \mathrm{C}, Z=6$, (b) $T=125.9^{\circ} \mathrm{C}, Z=7$, (c) $T=128.5^{\circ} \mathrm{C}, Z=8$, (d) $T=130.5^{\circ} \mathrm{C}, Z=9$, (e) $T=132.5^{\circ} \mathrm{C}, Z=10$ and (f) $T=135.2^{\circ} \mathrm{C}, Z=11$. The solid lines represent the measured spectra, where cell gap interference can clearly be observed. Dashed lines depict calculations according to (6) considering the birefringence dispersion, and dotted lines represent the calculations without consideration of the dispersion of birefringence, (2). 
should be small $\left(\approx 0.1 \mathrm{~K} \mathrm{~min}^{-1}\right)$, to reduce temperature hysteresis.

In Fig. 3 the temperature dependence of the cholesteric pitch $P$ is depicted as determined by this method $[10,11]$. The validity of the colour change method was verified by measurements according to the well established Cano-method [16]. To investigate the wavelength dependence of the optical rotation, the transmission spectra for the different twist states $Z=1,2,3 \ldots$ were recorded by an optical multichannel analyzer in combination with a polarization microscope equipped with a hot stage. For the measurements, very well oriented, defect free samples in Grandjean orientation have to be used. For the calculations of the birefringence dispersion according to (3), the value of $\lambda^{*}$ was determined independently. The UV spectrum of compound (S,S) M96, dissolved in chloroform, was recorded with a two beam spectrophotometer (Perkin-Elmer Lambda 15 UV/VIS Spectrophotometer). The mean wavelength was determined to $\lambda^{*}=267 \mathrm{~nm}$, the absorption band of the phenyl ring and the biphenyl group [18] (Figure 4). The value of $\bar{n}=1.51$ was obtained from interference spectra [19].

\section{Experimental Results and Discussion}

Figure 5 schematically depicts a spectrum between crossed polarizers calculated according to (6). The wavelength of the selective reflexion $\lambda_{0}$ was taken to be $5000 \mathrm{~nm}$, which corresponds to a cholesteric pitch of $P=3.3 \mu \mathrm{m}$, a typical experimentally observed value. The spectra were experimentally recorded in the visible range of the spectrum $(350-800 \mathrm{~nm})$ far from the region of the selective reflexion. These spectra are depicted in Fig. $6(\mathrm{a}-\mathrm{f})$ as the solid line for different twist states with $Z=6,7,8,9,10,11$, respectively. A shift in the maxima and minima of transmitted intensity is clearly observed in the temperature range considered. It should be noted that the spectra with $Z<6$ can only qualitatively be calculated with (6), a quantitative description being not satisfactory. This is most likely due to a slightly distorted cholesteric structure which deviates from the ideal form ((1), Fig. 2), as also a variation in intensity was observed when rotating the sample between crossed polarizers. Furthermore, the de Vries equation (2) was derived for an infinite helical structure. For the twist states $Z \geq 6$, the transmission char-

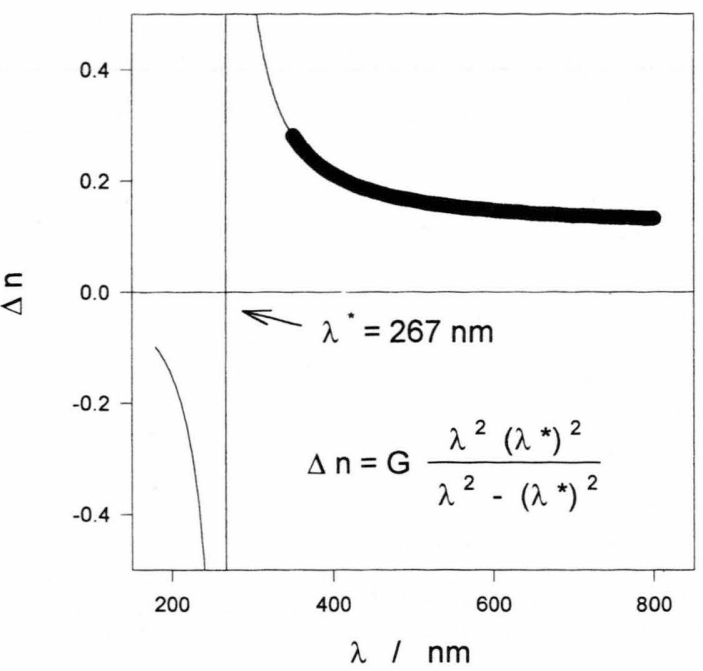

Fig. 7. Birefringence as a function of wavelength in the visible range of the spectrum, according to (3) with $G(T)=$ $1.65 \times 10^{12} \mathrm{~m}^{-2}$ and $\lambda^{*}=267 \mathrm{~nm}$.

acteristic can very well be modelled with (4), as depicted by the dashed lines in Fig. $6(\mathrm{a}-\mathrm{f})$, whereas a comparison of the calculations according to (2) (depicted as dotted lines) gives only poor agreement. For the presented calculations the generally temperature depend factor $G(T)$ can be taken constant, as $G(T)=$ $(1.65 \pm 0.05) \times 10^{12} \mathrm{~m}^{-2}$, because the temperature range investigated was far from the clearing point, and therefore changes of the nematic order parameter are negligible. The birefringence dispersion can then be determined by (3) and is depicted in Figure 7. It is clear that for a quantitative description of the optical properties of cholesterics, the birefringence dispersion has to be included.

\section{Conclusion}

The method of discontinuous colour changes of cholesteric samples in thin liquid crystal cells represents an additional method of pitch determination far from the selective reflexion. This method needs only very little substance $(\approx 5 \mathrm{mg})$, and the sample can further be used for other experiments in the same cell. It is necessary for a quantitative description of the optical properties to consider the birefringence dispersion 
I. Dierking et al. · Optical Properties of Cholesteric Phases

in the visible range of the spectrum. On the other hand, the method of discontinuous colour changes of cholesteric samples may also be used to detemine the birefringence dispersion by modelling the transmis-

[1] H. de Vries, Acta Cryst. 4, 219 (1951).

[2] M. F. Vuks, Opt. Spektrosk. 20, 644 (1966).

[3] S. T. Wu, Phys. Rev. A 33, 1270 (1986).

[4] S. T. Wu, J. Appl. Phys. 69, 2080 (1991)

[5] S. T. Wu, J. D. Margerum, H. B. Meng, L. R. Dalton, C. S. Hsu, and S. H. Lung, Appl. Phys. Lett. 61, 630 (1992).

[6] S.T. Wu, C. S. Wu, M. Warenghem, and M. Ismaili, Optical Engineering 32, 1775 (1993).

[7] W. Maier and A. Saupe, Z. Naturforsch. 14a, 882 (1959).

[8] W. Maier and A. Saupe, Z. Naturforsch. 15a, 287 (1960).

[9] I. Dierking, F. Gießelmann, P. Zugenmaier, K. Mohr, H. Zaschke, and W. Kuczynski, Liq. Cryst., in press.

[10] I. Dierking, F. Gießelmann, P. Zugenmaier, K. Mohr, H. Zaschke, and W. Kuczynski, Z. Naturforsch. 49a, 1081 (1994).

[11] I. Dierking, F. Gießelmann, and P. Zugenmaier, Mol. Cryst. Liq. Cryst., in press. sion spectra of planar Grandjean textures between crossed polarizers.

This work was supported by a grant from the Deutsche Forschungsgemeinschaft.

[12] A. Wiegeleben, L. Richter, J. Deresch, and D. Demus, Mol. Cryst. Liq. Cryst. 59, 329 (1980).

[13] L. Richter, D. Demus, and H. Sackmann, Mol. Cryst. Liq. Cryst. 71, 269 (1981).

[14] Y. Kamiishi, S. Kobayashi, and S. Iwayanagi, Mol. Cryst. Liq. Cryst. 67, 125 (1981).

[15] A. Wiegeleben and D. Demus, Cryst. Res. Technol. 17, 161 (1982).

[16] R. Cano, Bull. Soc. Fr. Miner. Cristallogr. 91, 20 (1968).

[17] F. Grandjean, C. R. Hebd. Seanc. Acad. Sci., Paris 172, 71 (1921).

[18] See for example: M. Hesse, H. Meier, and B. Zeeh, Spektroskopische Methoden in der organischen Chemie, Georg-Thieme-Verlag, Stuttgart 1984.

[19] W. Kuczynski and B. Stryla, Mol. Cryst. Liq. Cryst. 31, 267 (1975). The value of $\bar{n}=1.51$ was determined by W. Kuczynski and is in accordance with our measurements in [10]. 